\title{
Design of High Voltage Power Cable Real-time Monitoring System Function
}

\author{
XilinZhang ${ }^{1, ~ a, ~ D a c h u a n ~}$ Li $^{1, b}$, ZhenhaoWang $^{2, c}$ \\ ${ }^{1}$ State Grid Jilin Electric Power Company Limited Changchun Power Supply Company, Changchun, \\ Jilin Changchun 130021, China \\ ${ }^{2}$ Northeast Dianli University, Jilin Jilin 132012, China \\ aemail: xlz5859@sina.com, bemail: 609578551@qq.com, email: zhenhaowang@126.com
}

Keywords: High Voltage PowerCables; Fire Protection; Grounding Current; Lightning Arrester; Partial Discharge; Audio and Video; Real-time Monitoring

\begin{abstract}
This articlediscusses the high-voltage power cables feature real-time integrated monitoring system designed from the fire, earth currents, surge arresters and partial discharge monitoring and supervision of $3 \mathrm{G}$ remote audio and video transmission and other aspects. The Actual operating condition of a high-voltage power cables indicates the method of the necessity and urgency.
\end{abstract}

\section{Introduction}

Many failures will show the power cable to produce a ground sensor circulation, such as the metal sheath insulation damage, power cable metal sheath grounding system defect or failure. When the primary insulation quality changes, itwill be also reflected in the changes induced power metal sheath. Therefore, the effective monitoring for ground induced circulation can not only monitor power cable metal sheath own state, but also monitor many types of failures of the quality of the state's main insulation, can effectively prevent accidents, prolong the service life of power cables, ensure that the power cable safe and stable operation.

With the growth of lightning arrester operation time and the defect of its products have existed, lightning arrester aging at operating voltage and the damp problem is outstanding day by day. For lightning arrester, because of its speed of fault develops quickly, the traditional prevention test once a year is not completely find defects, and must be combined with other test means to master the operation of the arrester to ensure its safe operation.

Partial discharge monitoring is an effective method for the analysis of XLPE cable insulation deterioration degree, which is an urgent requirement for XLPE cable line partial discharge detection in order to achieve the status of their maintenance.

The current cable inspectionmainly rely on artificial regular patrol work, which mainly checks the circuit equipment running status and changes in environmental conditions along the line, the night patrol check line contacts are no fever flint, the phenomenon such as whether the insulator discharge and so on.

The current patrol of heavy workload, often appear leak and fault detection. There is also a big difficulty in management, which is a hidden danger to the safe operation of cable. Cable line emergency 3G remote video and audio transmission supervision system can effectively improve the emergency treatment effect of cable inspection and fault hidden trouble.

\section{The overall design idea}

According to the actual situation, the system designis based the concept of active monitoring and prevention [1], the use of advanced on-line monitoring technology, sensor technology, wireless communication technology, the electromagnetic induction to take energy technology, GIS technology and so on. To in-depth study of the failure mechanism of the cable, the realization of online monitoring and analysis of high voltage power cable [2]. An in-depth study [4] on failure 
mechanism of cable can realize on-line monitoring and analysis of high voltage power cable [3].

- Based on the on-line monitoring technology and the advanced sensor technology;

- Using a new technology to solve the technical problem of power supply of high voltage side of electronic device. Designing the key circuit of super capacitor based on electromagnetic induction method, which has instant high power supply, storage, low temperature, environmental protection, simple maintenance etc;

- Using solar energy technology to provide power foroverhead line collection device;

- Based on wireless communication technology(GSM/GPRS)datatransmission;

- GIS technology, data compression and control center management system storage technology, multi threading technology, the wireless communication based management, the monitoring center management system of wireless communication management;

- With the cable joint temperature, the insulating sheath grounding current, lightning arrester leakage current, partial discharge and integrated audio and video monitoring function.

\section{High voltage power cable fire safety remote online monitoring}

Installing a cable joint wireless temperature sensor in the cable connector can monitor the joint temperature. The temperature of each cable joint was transferred to the corresponding cable temperature emonitoring data acquisition repeater through the way of wireless communication. Cable temperature monitoring data acquisition repeater is responsible for real-time collection of temperature data transmitted by each wireless temperature sensor. When the cable joint temperature exceeds the warning temperature, repeat erssend alarm signal to the computer monitoring and management control analysis system centerautomatically. The system can send alarm signal to the person in charge of the corresponding through the GSM short message according to actual requirement. The computer management system can interact data with the monitoring device through wireless communication and display the various cable joint temperature data in real-time. When the temperature eexceeds the warning value, the system sends out the alarm signal to inform the operating personnelto take appropriate measures to deal with. In addition, the computer management system judges the running status of cable joint through the analysis of the joint of the historical temperature data, which is for better prevent the occurrence of accidents.

\section{Ground current online monitoring is connected with the high voltage power cable}

According to the actual situation, the grounding induced current was existed in power cables. The ground induction current includes grounding capacitance current and GICC.

Metal protecting layer circulation size is mainly determined by EMF, loop impedance. Single core cable metal sheath layer induced potential is not only dependent on the cable phase voltage and load, but also depends on the cable arrangement mode and the length of line, even are related with the presence of reflux, reflux line number. The loop impedance is also associated with the geographical environment of cable laying and ground properties. Therefore, the research process theory is more complexon circulation method calculation. In order to reduce the cable line loss and improve the cableconveying capacity, short line single core cables [2] are used for end is directly connected to the other end protection and grounding mode. The cross connection grounding mode is used for the long line of single core cable.

\section{The line arrester of high voltage power cable on-line monitoring}

Every arrester grounding wire access the sensor monitoring of leakage current [3]. MOA was installed with the wireless communication module of temperature monitoring. Lightning arrester valve temperature data was sent to the data acquisition to handle through wireless radio frequency communication. All monitoring data was sent to the monitoring and management terminal. In order to save resources, each phase of leakage current and a valve sheet temperature data through wireless radio frequency communication flows into $\mathrm{B}$ phase data acquisition device. The $\mathrm{B}$ phase device 
monitoring terminal is unified through the computer management system of GSM network to the remote monitoring analysis. Monitoring terminal receives the real-time data and manages multiple data acquisition device, and connects remote computer system through the GSM/GPRS network communication. Monitoring terminal receives the data command from the monitoring center, monitoring center can set the clock terminal unit, the SIM card number of monitoring center and alarm limit terminal parameters by the short message. Terminal send alarm signal to the monitoring center PC automatically when it is more than vigilance parameters, and alarm messages can be automatically forwarded to the cable maintenance personnel.

\section{Online monitoring of partial discharge in high voltage power cable}

In the method of pulse detection of cable fault location, various kinds of disturbances exist inevitably. According to the time domain characteristics, disturbance can be divided into three categories: periodic, narrowand disturbances, white noise disturbance and pulse disturbance. Among them, white noise disturbance includes all kinds of random noise, such as winding thermal noise, ground noise and thermal noise of measuring instruments, etc. Due to white noise spectrum and partial discharge signal spectrum are similar, so it is difficult to use traditional Fourier analysis method to filter it. So we must adopt effective digital signal processing method [4] to eliminate the influence of these disturbances and improve the positioning accuracy.

The emergence of the wavelet analysis method [4] has opened up a new idea for the inhibition of white noise. Wavelet analysis is a kind of analysis method s of signal time - scale (time - frequency), which has the characteristics of multi-resolution analysis. More importantly, it has the ability of denoting local signal characteristics in both frequency domain and time domain, namely Higher frequency resolution and Lower time resolution in the low-frequency part and instead in the high-frequency, which is quite fit for detecting normal signal.

Modulus maximum of wavelet transform is a concentrated reflection of the signal singularity. There is completely different properties between the behavior of white noise and The singularity of the signal in wavelet transform, namely Modulus maximum of wavelet transform generated by the noise signal decreases with increasing scale, while the other Modulus maximum of wavelet transform generated by other signals increases with increasing scale(for step signal remains the same). On the basis of that we can eliminate noise effectively.

\section{G Remote audio and video transmission supervision system}

Using 3G Wireless communication technology, image compression technology, GIS technology to complete the cable line operations management and emergency treatment of $3 \mathrm{G}$ remote video and audio transmission monitoring system, and using the theory of agglomeration energy of the wavelet algorithm and the separation technology of the Background target to put the practical model of the wavelet algorithm which consist by multilevel tree groups, a generalized wavelet, mathematical morphological wavelet and so on embedded application to the project, complete NVCT video compression technology [5]. The system is divided into individual system and vehicle system to interaction use, realize the operational inspection of the cable line patrol, personnel positioning, and emergency treatment to the hidden fault and cable line long distance emergency command, and other functions. Mastering the operation of the cable line and equipment, and found the hidden fault of the line timely, improve the safety and reliability of operation of the power supply.

\section{Conclusion}

The four high voltage power cables on-line monitoring subsystem has been put into field operation from fire protection, grounding current, lightning arrester and partial discharge, which has achieved good effect. The 3G remote audio and video transmission supervision systems are being developed, which accumulated valuable experien cefor the development of real-time comprehensive monitoring system of high voltage power cables. 
The gradually increase of the urban power supply network cabling rate and the power supply reliability requirements of the city supply power network, requirements power grid operation maintenance management department master the health status of high voltage power cables in real-time, it's very necessary and urgent to explore the high voltage power cables real-time monitoring system.

\section{References}

[1] Xilin Zhang, ZhenhaoWang,Yechun Xin.Overall design of Changchun grid power cables fireproof remote online monitoring system [J].CARPI. 2012.105-109.

[2] Xilin Zhang, Zhenhao Wang, Guoqing Li.The Research and Development of the On-line Monitoring System for Grounding Current of High-voltage Cable [J].APPEEC. 2012.

[3]ZhenhaoWang, XilinZhang, XiaojuanHan.Research and Development on the On-line Monitoring System of Cable Line Arrester [J].REET. 2013.2294-2297.

[4] DanPang,XilinZhang,ZhenhaoWang.Functions and Theoretical Basis of Partical Discharge On-line Monitoring System for High Voltage Cables [J].REET. 2014.

[5] Yuanping Liu, Jingliang He, Hongxin Wang. Discussion on the on-line monitoring method of the XLPE cable.[J]. High Voltage Technology, 2001 27(4)26-27. 